\title{
sciendo
}

10.2478/AMB-2019-0003

\section{COST-MINIMIZATION ANALYSIS OF NON-INVASIVE AND INVASIVE MECHANICAL VENTILATION FOR DE NOVO ACUTE HYPOXEMIC RESPIRATORY FAILURE IN AN EASTERN EUROPEAN SETTING}

\author{
V. Ilieva ${ }^{1}$, T. Mihalova ${ }^{2}$, Yo. Yamakova ${ }^{1}$, R. Petkov ${ }^{2}$, B. Velev ${ }^{1}$ \\ ${ }^{1}$ Anaesthesiology and Intensive Care, University Hospital of Pulmonary Diseases, Medical University - Sofia, Bulgaria \\ ${ }^{2}$ First Therapeutic Clinic, University Hospital of Pulmonary Diseases, Medical University - Sofia, Bulgaria
}

\begin{abstract}
Introduction: In the light of constant pressure for minimizing healthcare costs we made a cost-minimization analysis comparing invasive mechanical ventilation (IMV) and non-invasive ventilation (NIV) as treatment for hypoxemic acute respiratory failure (ARF). Aim: The primary objective was to estimate the direct medical costs generated by a patient on IMV and NIV. A secondary objective was to identify which aspect of the treatment was most expensive. Material and Methods: This is a single center retrospective study including 36 patients on mechanical ventilation due to hypoxemic ARF, separated in two groups - NIV $(n=18)$ and IMV $(n=18)$. We calculated all direct medical costs in Euro and compared them statistically. Results: On admission the $\mathrm{PaO}_{2} / \mathrm{FiO}_{2}$ and SAPS II score were comparable in both groups. We observed a significant difference in the costs per patient for drug treatment (NIV: 616.07; IQR: 236.68, IMV:1456.18; IQR:1741.95, $p=0.005$ ), consumables (NIV: 16.47; IQR: 21.44, IMV: 98.79; IQR: 81.52, $p<0.001$ ) and diagnostic tests (NIV: 351; IQR: 183.88, IMV: 765.69; IQR: 851.43, $p<0.001)$. We also computed the costs per patient per day and there was a significant difference in the costs in all above listed categories. In both groups the highest costs were for drug treatment - around $61 \%$. Conclusions: In the setting of hypoxemic ARF NIV reduces significantly the direct medical costs of treatment in comparison to IMV. The decreased costs in NIV are not associated with severity of disease according to the respiratory quotient and SAPS II score.
\end{abstract}

Key words: non-invasive ventilation, invasive ventilation, mechanical ventilation, pharmacoeconomy, cost-minimization

Corresponding author: Viktoria llieva, University Hospital of Pulmonary Diseases, Medical University - Sofia, Bul. "Acad. Ivan Evstatiev Geshov" 19, Sofia, Bulgaria 1431, tel.: +359/ 889907202 , e-mail: vicktoria.ilieva@gmail.com

\section{INTRODUCTION}

on-invasive ventilation (NIV) is now a worldwide standard of care for hypercapnic acute respiratory failure (ARF) but its use in hypoxemic ARF is controversial, mainly because of lack of strong evidence, coming from randomized controlled trials. In the official European Respiratory Society/ American Thoracic Society clinical practice guidelines from 2017 the task force does not make any recommendation regarding the use of NIV on this indication [1]. 
There are a lot of clinical trials regarding NIV in the acute setting. However, only a few studies with heterogeneous quality discuss pharmacoeconomical issues, most of which include only COPD patients [2].

Hospital directors and The National Health Care Fund in Bulgaria are very concerned about health care expenditures and the amount of money spent on health care services. In the light of constant pressure for minimizing medical costs from healthcare managers all over Eastern Europe we made a cost-minimization analysis comparing IMV and NIV as treatment methods for acute hypoxemic respiratory failure.

Aim: The main objective of our study is to estimate the direct medical costs generated by a patient on IMV and NIV during their Intensive Care Unit (ICU) stay. A secondary objective is to identify which aspect of the treatment is most expensive.

\section{MATERIAL AND METHODS}

This study includes 36 patients, requiring mechanical ventilation for de novo hypoxemic ARF due to severe pneumonia and admitted to the medical ICU of National Hospital for Pulmonary Diseases "Sv. Sofia" during the period 2015-2017. The diagnosis severe pneumonia was based on the criteria of the Infectious Diseases Society of America/American Thoracic Society [3].

The two groups in our study were defined by method of ventilation - IMV $(n=18)$ and NIV $(n=18)$. Which of the two treatment methods chosen was based on the clinicians' opinion, supported by existing NIV guidelines during the study period [4].

Two types of data were extracted from the hospital files: information regarding the severity of the disease $\left(\mathrm{FiO}_{2} / \mathrm{PaO}_{2}\right.$, respiratory rate and SAPS II score) and direct medical costs (for drug treatment, con- sumables and diagnostic tests). Costs for human resources, capital equipment, and overheads were not included, because we assumed that they were comparable for all patients as they were treated in the same ICU.

First we computed the costs for every patient and compared them by groups. Then we calculated costs per patient per day and compared them again.

All prices were extracted from the official 2018 price list used in University Hospital of Pulmonary Diseases, Sofia, Bulgaria. The costs are reported in Euro $(€)$ according to the local exchange rate on 04.08.2018.

The statistical analysis was conducted with the IBM SPSS v. 25, using Mann Whitney U-test. All the results are presented as median and inter quartile range (IQR).

\section{RESULTS}

On admission the $\mathrm{PaO}_{2} / \mathrm{FiO}_{2}$ (median: 135.02, IQR: 32.44 vs. median: 127.5 , IQR: $23.73, p=0.07$ ), respiratory rate (median: 31.44 , IQR: 10.11 vs. median: 29.55, IQR: 3.9, $\mathrm{p}=0.92$ ) and SAPS II score (median: 37.9, IQR: 18.47 vs. median: 30.9, IQR: 10.34, $\mathrm{p}=0.06$ ) were comparable in both groups.

We observed a statistically significant difference in the overall costs per patient for drug treatment, consumables and diagnostic tests (Table 1). The highest costs were for drug treatment $-60.89 \%$ in the IMV and $61.52 \%$ in the NIV group (Fig. 1).

The costs per patient per day were also computed and there was again a significant difference in all defined categories (Table 2).

We payed special attention to antibiotics as a part of general drug treatment costs. In our sample antibiotics were $31.3 \%$ in the IMV as compared to $28.33 \%$
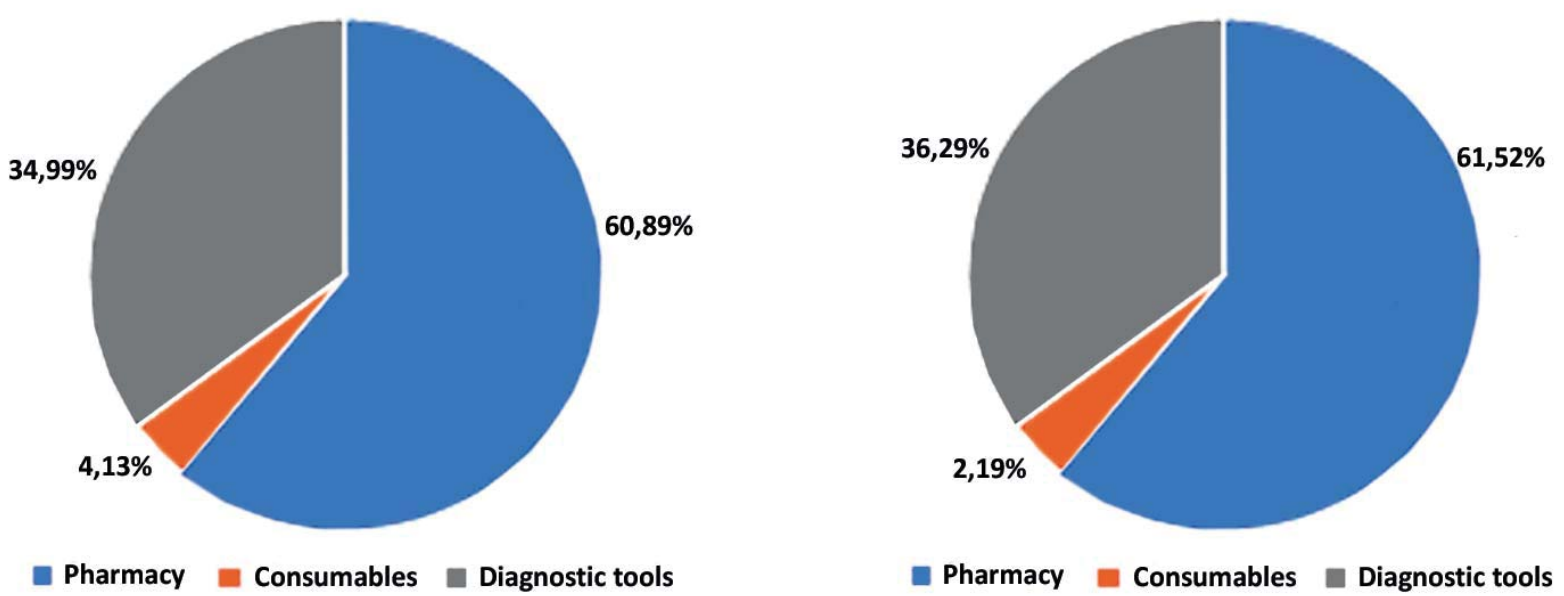

Fig. 1. Graphic presentation of the direct medical costs in the IMV (left) and NIV (right) groups 
in the NIV group. There was no significant difference between the antibiotic costs per patient but it became significant when we made a per patient per day calculation (Table 1 and 2).

Table 1. Comparison of the costs per patient between the IMV and NIV groups (in €)

\begin{tabular}{|l|l|l|l|}
\hline & IMV $(\mathbf{n}=18)$ & NIV $(\mathbf{n}=18)$ & $\mathbf{p}$ \\
\hline Drug treatment & Median: 1456.18; IQR:1741.95 & Median: 616.07; IQR: 236.68 & 0.005 \\
\hline Antibiotics & Median: 358.22; IQR: 620.93 & Median: 151.79; IQR: 96.69 & 0.2 \\
\hline Consumables & Median: 98.79; IQR: 81.52 & Median: 16.47; IQR: 21.44 & $<0.001$ \\
\hline Diagnostic tests & Median: 765.69; IQR: 851.43 & Median: 351; IQR: 183.88 & $<0.001$ \\
\hline Overall & Median: 2246.91; IQR: 2471.35 & Median: 954.76; IQR: 370.42 & $<0.001$ \\
\hline
\end{tabular}

Table 2. Comparison of the costs per patient per day between the IMV and NIV groups (in €)

\begin{tabular}{|l|l|l|l|}
\hline & IMV $(\mathbf{n}=\mathbf{1 8})$ & NIV $(\mathbf{n}=18)$ & $\mathbf{p}$ \\
\hline Drug treatment & Median: 75,22; IQR: 28,61 & Median: 46,03; IQR: 11,14 & $<0.001$ \\
\hline Antibiotics & Median: 16,16; IQR: 12,27 & Median: 13,15; IQR: 5,52 & 0.04 \\
\hline Consumables & Median: 5,13; IQR: 5,17 & Median: 1,32; IQR: 1,56 & $<0.001$ \\
\hline Diagnostic tests & Median: 41,54; IQR: 40,51 & Median: 26; IQR: 12,71 & $<0.001$ \\
\hline Overall & Median: 122,16; IQR: 72,91 & Median: 75,98; IQR: 26,5 & $<0.001$ \\
\hline
\end{tabular}

duces the rate of nososcomial pneumonia and therefore the use of antibiotics [13,14]. It is possible that we did nor encounter any statistical significance in this subcategory because all our patients suffered from a primary pulmonary infection and we had to use a full set of antibiotics in their treatment regimen.

Establisaling significant cost reduction in the NIV compared to the IMV group we also computed the costs per patient per day to avoid any bias regarding the hospital stay length. The obtained results after this second analysis remained significant.

A limitation of our study is that we measured only the direct medical costs and did not include human resources. In the beginning NIV can be very time consuming for the personnel [15]. But in our experience after proper training nurses spend less time for a patient on NIV, than on IMV. Another limitation of this study is the small sample size and its retrospective nature. We will continue to collect data prospectively and

\section{DISCUSSION}

We conducted a literature search on the pharmacoeconomy of NIV and hypoxemic ARF, and we did not find any related articles. Most similar were several cost-effectiveness analyses of NIV for COPD exacerbations and acute cardiogenic pulmonary edema. Most of them are ICU based and compared NIV with IMV or standard therapy, but some had studied NIV in the medical ward. A clear reduction of medical costs and enhanced efficacy was noted in all of them [5-12]. To our knowledge, we present the first pharmacoeconomical analysis regarding patients treated with NIV and IMV for hypoxemic ARF (excluding cardiogenic pulmonary edema). We defined three categories of direct medical costs - drug treatment (including oxygen), consumables and diagnostic tests. After conduction of statistical analysis, we found a significant difference between the costs in all categories between the two defined study groups (IMV and NIV). Highest were the costs for drug treatment, around $60 \%$ of which were for antibiotics. Although we expected to see a difference in the antibiotic costs, we could not report one. Clinical trials show that NIV re- make another analysis to reconfirm our findings.

Conclusions: We have shown that the direct medical costs for a patient with hypoxemic ARF on NIV are significantly less than those for a patient suffering the same condition and placed on IMV. This difference is maintained even after correction for the length of hospital stay of each patient (costs per patient per day). In both groups the highest costs were for drug treatment. Therefore, we can conclude that for patients with hypoxemic ARF in an ICU NIV is cheaper than IMV.

\section{Conflict of interests: None declared}

Acknowledgements: None

\section{REFERENCES}

1. Rochwerg B, Brochard L, Elliott MW, et al. Official ERS/ATS clinical practice guidelines: noninvasive ventilation for acute respiratory failure. Eur Respir J. 2017;50:1602426.

2. Nicolini A, Stieglitz S, Bou-Khalil P, Esquinas A. Cost-utility of non-inasive mechanical ventilation: Analysis and implications in acute respiratory failure. A brief narrative review. Respr Investig. 2018;56(3):207-13. 
3. Mandell A, Wunderink R, Anzueto A et al. Infectious Diseases Society of America/American Thoracic Society Consensus Guidelines on the Management of Community-Acquired Pneumonia in Adults. Clin Infect Dis. 2007;44:S27-72.

4. British Thoracic Society Standards of Care Committee. BTS guideline: Non-invasive ventilation in acute respiratory failure. Thorax. 2002;57:192-211.

5. Nava S, Evangelisti I, Rampulla $\mathrm{C}$ et al. Human and financial costs of noninvasive mechanical ventilation in patients affected by COPD and acute respiratory failure. Chest. 1997;111(6):1631-8.

6. Keenan SP, Gregor J, Sibbald WJ et al. Noninvasive positive pressure ventilation in the setting of severe, acute exacerbations of chronic obstructive pulmonary disease: more effective and less expensive. Crit Care Med. 2000;28(6):2094-102.

7. Criner GJ, Kreimer DT, Tomaselli $M$ et al. Financial implications of noninvasive positive pressure ventilation (NPPV). Chest. 1995; 108:475-82.

8. Plant PK, Owen JL, Parrott S, Elliot MW. Cost effectiveness of ward based non-invasive ventilation for acute exacerbations of chronic obstructive pulmonary disease: economic analysis of randomised controlled trial. Br Med J. 2003;326(7396):956.

9. Patel SP, Pena M, Babcock Cl. Cost effectiveness of noninvasive ventilation for chronic obstructive pulmonary disease related respiratory failure in Indian hospital without ICU facilities. Lung India. 2015;32(6):549-56.

10. Ornek $\mathrm{T}$, Tor $\mathrm{M}$, Altin $\mathrm{R}$ et al. Clinical factors affecting the direct cost of patients hospitalized with acute exacerbation of chronic obstructive pulmonary disease. Int $\mathrm{J}$ Med Sci. 2012;9(4):285-90.

11. Bertolini G, Confalonieri M, Rossi $C$ et al. Costs of the COPD. Differences between intensive care unit and respiratory intermediate care unit. Respir Med. 2005;99:894-900.

12. Chandra K, Blackhouse G, Campbell M, Costa KV. Cost-effectiveness of interventions for chronic obstructive pulmonary disease (COPD) using an Ontario policy model. Ont Health Technol Assess Ser. 2012;12(12):1-61.

13. Girou E, Schortgen F, Delclaux C et al. Association of noninvasive ventilation with nosocomial infections and survival in critically ill patients. JAMA. 2000;284(18):2361-7.

14. Nourdine $K$, Combes $P$, Carton MJ et al. Does noninvasive ventilation reduce the ICU nosocomial infection risk? A prospective clinical survey. Intensive Care Med. 1999;25(6):567-73.

15. Chevrolet JC, Jolliet P, Abajo B et al. Nasal positive pressure ventilation in patients with acute respiratory failure. Difficult and time-consuming procedure for nurses. Chest. 1991;100(3):775-82. 